\title{
A comparison study of processing and visualization in image analytics
}

\author{
C. Jittawiriyanukoon* \\ Graduate School of Advanced Technology Management, Assumption University, Bang Sao Thong District, Samut \\ Prakan Province, Thailand
}

Received: 28-December-2019; Revised: 31-January-2020; Accepted: 10-February-2020

(C)2020 C. Jittawiriyanukoon. This is an open access article distributed under the Creative Commons Attribution (CC BY) License, which permits unrestricted use, distribution, and reproduction in any medium, provided the original work is properly cited.

\begin{abstract}
Image analytics is a process to evaluate and mine the insight about digital images. The emergence of QR-code-technology is a case, but practical cases include face as well as text recognition and analysis. This article explores smoothing approaches to enrich an image by filtering negative elements and stressing positive ones. Image smoothing is a common process and significant in mining digital images. The paper strengthens an appropriate algorithm for sharpening images by applying linear and non-linear algorithms. Sub-standardized and degraded images need to be previously sharpened prior to computer vision. It is found that the average smoothing algorithm technique outperforms other approaches regarding noise filtering as it focuses on static window size and computes the edges. Besides, it is also simply carried out in computation.
\end{abstract}

\section{Keywords}

Computer vision, Frequency domain, Image analytics, Image processing, Visualization.

\section{Introduction}

A digital image is a mathematical dataset of a $2 \mathrm{D}$ function. It is a raster image represented by pixels [1]. To process digital image analytics, we have to develop numerical values describing discrete elements of the image. This numerical analysis may be further performed by a machine. The conversion of image data (big data) to $2 \mathrm{D}$ representation $(\mathrm{F}(\mathrm{x}, \mathrm{y}))$ will be executed to reflect the original intensity of the image [2]. The execution of image analytics is extensively practical. Many places comprehend how to separate value from image data to construct different knowledge sources in traffic analysis, computer network security and transportation safety [3]. Vasanth and Nataraj [4] are curious to recognize facial actions and features from images in computer vision. It can be applicable for real-time video to match with recorded face images (image recognition). The research helps make image analytics happen due to deep learning application, big data analytics and matured technology.

\footnotetext{
*Author for correspondence
}

The authors also point out that various practical applications like animation involve with recognition technology. Support Vector Machine (SVM) algorithm is obtained to categorize the action and appearance (emotion) out of facial pattern recognition.

The smoothing process is to ease unwanted elements (noise) and enhance features of the image graphic. Both non-linear [5] and linear [6] algorithms are functional for smoothing digital images. To smooth the image helps comfort multiple processes in image analytics. Linear smoothers are techniques that involve with a pixel intensity, which gives the linear sum amount in a sliding window. It is correspondent to the Fourier transform by multiplying and integrating for each image. Let a linear model denote as $y i=$ aix, where $x$ identifies the unknown variables with a (j x 1) matrix, ai represents an (i x j) 2D matrix regarding input parameters from original image, and yi contains the ( $\mathrm{i} x \mathrm{1}$ ) matrix of observed output. Regarding this recursive computation, $i$ input parameters are employed with $\mathrm{j}$ unknown variables and each iteration results $i$ observed outputs. 
With,

$y_{i}=\left[\begin{array}{c}y_{1} \\ y_{2} \\ \vdots \\ y_{i}\end{array}\right], a_{i}=\left[\begin{array}{ccc}a_{11} & \ldots & a_{1 j} \\ a_{21} & \ldots & a_{2 j} \\ \vdots & \ddots & \vdots \\ a_{i 1} & \ldots & a_{i j}\end{array}\right]$, and $x_{i}=\left[\begin{array}{c}x_{1} \\ x_{2} \\ \vdots \\ x_{j}\end{array}\right]$.

Non-linear systems [7] have diversified transform compared to linear systems. They are techniques that cannot be only transformed by either convolution or Fourier concept. A sliding average value is a model of a non-linear one. For non-linear smoothers, the response or output of the recursion does not follow varying concurrence. Several actual problems exhibit nonlinear smoothing function which involves adjustments to linear smoothers. The non-linear smoothers can be formulated in a state-space domain, and the smoother estimates by associating linearization at each recursive operation [8]. It means adjusting interpolation to the observed curves at the current time step in order that the typical smoother recursions can be applied in parallel to harvest approximations for the next time step. Besides, nonlinear smoother is following the score function with the input distribution from sources, it is possible to approximate adaptively the source convolution by using corresponding least-squared calculation [9]. Bennasar, et al. [10] also explicitly approximate scoring functions for feature selection process. It is based on common features like the correlation with predictable variables. This approach outclasses other techniques on actual datasets, reduces the mean error about six percent, and results the outstanding tradeoff concerning stability and accuracy. One of nonlinear smoothing techniques is based on ordered parameters. It is referred to non-linear as it cannot use linearity like a convolution value. The ordered approach stretches all pixels in sequence and substitutes the pivot. The objective of this article is to explore smoothing approaches to enrich an image by filtering negative elements and stressing positive ones. Moreover, we can predict the computation cost as we smooth $\mathrm{n} x \mathrm{n}$ pixels in the case of insertion sort the average time complexity is $\mathrm{O}(\mathrm{n} 2)$ and the space complexity is $\mathrm{O}(1)$. In the case of merge sort the average time complexity is $\mathrm{O}(\mathrm{n} \log \mathrm{n})$ but the space complexity is $\mathrm{O}(\mathrm{n})$.

\section{Noise patterns}

The Currently, valuable information can be mined from digital images, for example, biometric images and medical images like X-ray. Since the insight from digital images is more tolerant to be extracted compared to telecommunication signal de-noising [11], digital images are trivial research. However, digital images are often accidentally defected by noise. Image is a dominant source to display visualization. In image processing, smoothing is commonly recommended. TV images are degraded by noise [12] during the broadcast due to interference and poor receptor. Noise is also induced in digital creations [13] after scanning. In real-world environment, the study of image smoothing requires constant consideration of noise patterns [14]. In this regard, engineers have proposed reviews on both academic and applied aspects. Many articles recommend the noise in imaging processing present during image retrieval, encoding, and traversing steps [15]. The presence of noise corrupts the live information of the images. In this common logic as a researcher, we need to investigate how severe the current image is corrupted, which noise pattern is attended, and how we smooth. Occasionally, we may need to understand the logical and realistic patterns of noise associated with digital images. In this section, we address the key to these complications through the reflection of noise patterns. Noise symbolizes unpleasant part of the image and reflects the image value. Noise can be described as an unwanted element $(\mathrm{N})$ which disturbs the defected image (D). Assume that the actual image (A) is affected by noise pattern and is represented as:

$\mathrm{D}(\mathrm{m}, \mathrm{n})=\mathrm{N}(\mathrm{m}, \mathrm{n})+\mathrm{A}(\mathrm{m}, \mathrm{n})$

Noise originates from different sources and induces into images after scanning. The scan process transforms original image data into digital data. In each transformation step, it introduces noise at random position in which noise arises from normal sensations. Finally, these steps naturally outcome a convolution of actual pixels. Noise can be demonstrated by a probability density function (PDF) which lies over on the actual image. The most common patterns of noise are introduced: salt and pepper noise, Gaussian noise, Erlang noise and negative exponential noise.

\subsection{The salt and pepper noise}

It is a pattern of noise normally seen on digital images [16]. It is white and black pixels in a digital image at random spots. In this pattern, we can represent a digital image by eight bits per pixel and usually the intensity of salt noise is close to $(28-1)$ while pepper noise makes close to zero. Note that the probability of each noise fluctuation is lower than ten percent unless the noise could override the actual image. 


\subsection{Gaussian noise}

In a communication network, telecommunication channels can be induced by Gaussian noise deriving from regular sources, such as the heat, thermal objects, etc. The Gaussian noise follows a Gaussian PDF and in 2D, it can be calculated by the product of $\mathrm{x}$-domain and y-domain as shown in Equation 2.

$$
P D F_{G}(x, y)=\frac{1}{2 \pi \sigma^{2}} e^{-\frac{(x-\mu)^{2}+(y-\mu)^{2}}{2 \sigma^{2}}}
$$

Where $\mathrm{x}$ is the gray intensity in the $\mathrm{x}$-axis, $\mathrm{y}$ is the gray intensity in the $y$-axis, $\mu$ is the mean value, and $\sigma$ is the standard deviation of the Gaussian distribution. In practice, seventy percent of values fall in the range $\mu \pm \sigma$ and ninety percent of the same fall between $\mu \pm 2 \sigma$. Note that we assume $\mathrm{PDF}_{\mathrm{G}}$ is zeroed if it falls further than $\mu \pm 3 \sigma$. Gaussian noise is suitable to analyze a typical step which induces noise.

\subsection{Erlang noise}

Like other noise patterns, an Erlang-k distribution follows a particular PDF. The Erlang-k distribution has been promoted by to measure the users' traffic which can be requested concurrently to a switching node in computer network. Erlang-k's distribution has been commonly employed in queuing fundamental to analyze mathematically the waiting time in lines. It is also utilized in statistical analysis for biomedical steps, and in random process.

The equation of an Erlang-k PDF in one dimension as shown in equation 3 can be represented by two parameters, $\mu$ and $\mathrm{k}$, where:

- $\mathrm{k}$ must be a positive number and denotes the shape of an Erlang function.

- $\mu$ is an average service rate.

$P D F_{E_{k}}(x, \mu, k)=\frac{e^{\frac{-x}{\mu}} x^{k-1}}{\mu^{k}(k-1) !}$ for $\mu>0$ and $x \geq$

0 .

\subsection{The negative exponential noise}

Various noise patterns that induce in natural sensations follow a function of a negative exponential PDF. The negative exponential distribution is one of the critical distributions in queuing analysis. Engineer employs a negative exponential PDF to compute the strength of tension in civil engineering. An architect also utilizes it regularly to construct, design, and develop structures and machines. It allows an aeronautical engineer to simulate how aircraft completes during the flight. Negative exponential formula is employed to comprehend kinetic energy of the wave, and to calculate heat, pressure, and wind flow. Not to mention a sound engineer applies it to analyze wave patterns. To form a negative exponential PDF, we only use a parameter $\mu$ (an average service rate) in one dimension as shown in Equation 4.

$$
P D F_{N E}(x, \mu)=\frac{1}{\mu} e^{-\frac{x}{\mu}} \text { for } \mu>0 \text { and } x \geq 0
$$

\section{Smoothing algorithms}

The resolution of smoothing is to diminish noise for a better being visualization. Irregularly, smoothing can be specified as filtering. There are two smoothers that have been uncovered practical in image analytics, namely, a spatial smoother and a temporal smoother. The former one is applicable for both dynamic and static digital images. The latter one is valid only for dynamic objects. A spatial smoother is utilized for lessening the noise and concealing [17]. It is to decrease the level of image variance [18]. It can help eliminate some details like gaps between curves from the digital image. A smoothing procedure can start from improving $5 \times 5$ pixels. The modest way is to read all value exhibited in each pixel, even them out then substitute to the pivotal one. A computation keeps iterating for limited particular elements or the whole image pixels. We smooth $\mathrm{n} \times \mathrm{n}$ pixels with restricted portion of $\mathrm{i} x \mathrm{j}$ concerning the image size of $\mathrm{x}$ rows and $\mathrm{y}$ columns then the average time complexity is $O\left(n^{2} i j\right)$, where $i \in x$ and $j \in$

\subsection{Sobel smoothing alsgorithm (SSA)}

Edge in digital images is boundary with divergence of intensity - a descent in intensity from pixel to pixel. It reserves the significant possession in a considered image. The edge detection of a digital image is a key to filter out noise (useless information). Sobel algorithm [19] is investigated by employing toolbox's function. It estimates the descent to discover edges. It assumes edges whenever the gradient descent (GD) of the recursive calculation regarding the targeted image is achieved. In 2D spatial smoother, it refers to an $\mathrm{n} \times \mathrm{n}$ gradient matrix.

\subsection{Prewitt smoothing algorithm (PSA)}

There exists an edge detection algorithm, called Prewitt method [20]. The algorithm uses a discrete gradient estimator to calculate the differentiation of the intensity of considered image. At each recursion, the algorithm results the norm or the gradient of this vector. It depends on the image's convolution with a trivial, discrete, and gradient magnitude in two 
dimensions, horizontal and vertical axes. It is thus comparatively economical due to the average time complexity. Additionally, the calculation in the gradient estimation is also somewhat unsophisticated, especially in case of the digital image with high variance in the frequency domain

\subsection{Average smoothing algorithm (ASA)}

The ASA [21] based on linearity is windowed smoother which sharpens digital images. The algorithm functions as a low-pass smoother in signal processing. The straightforward computation behind the algorithm is for any pixels of considered image finding an average value across its vicinity

\subsection{Laplacian smoothing algorithm (LSA)}

The LSA [22] is a $2 \mathrm{D}$ recursive computation of the second derivative of considered image. It firstly marks an area of quick intensity variation and

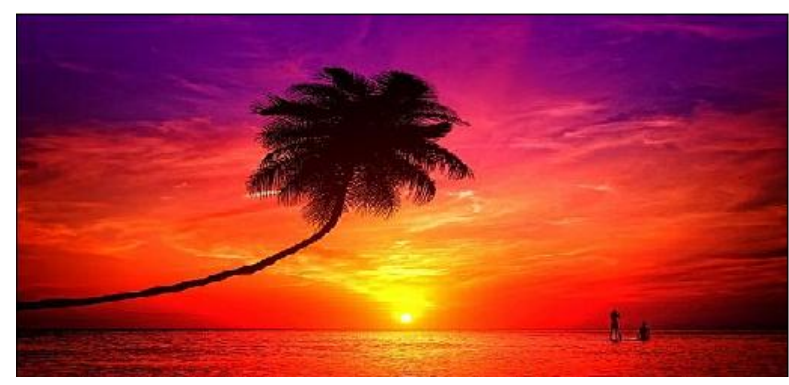

(a)

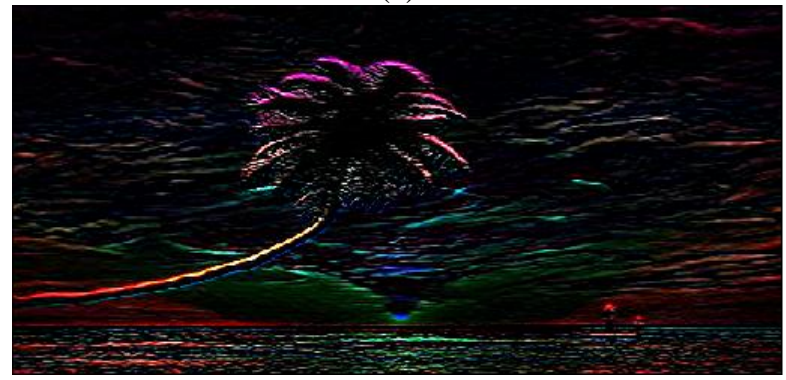

(c)

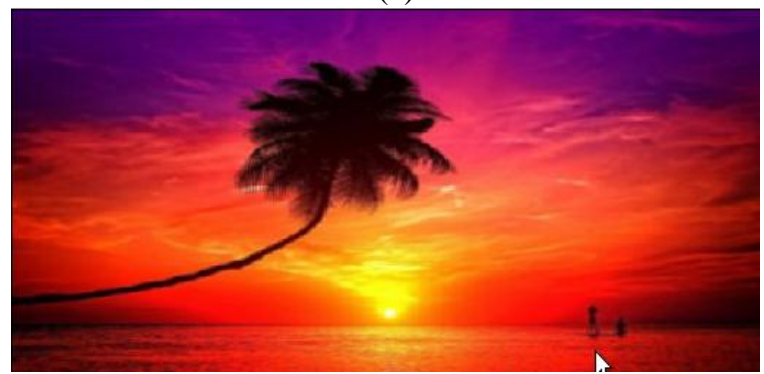

(e) highlights them as the edge estimation. The algorithm regularly employs to digital images that have been smoothed by some estimation techniques before. The additional process by LSA is to lower its vulnerability to noise.

The principal purpose in digital image smoothing [23] is to underline acceptable detail in the image, and to enrich detail that has been corrupted, because of movement effect or noise. Smoothing can be completed by spatial variation. Figure 1 displays the results of previously mentioned smoothers by comparing them to original and corrupted images. Note that in our evaluation of the above-mentioned smoothing algorithms, we limit all approaches regarding noise filtering by focusing on only static window size to calculate the edges.

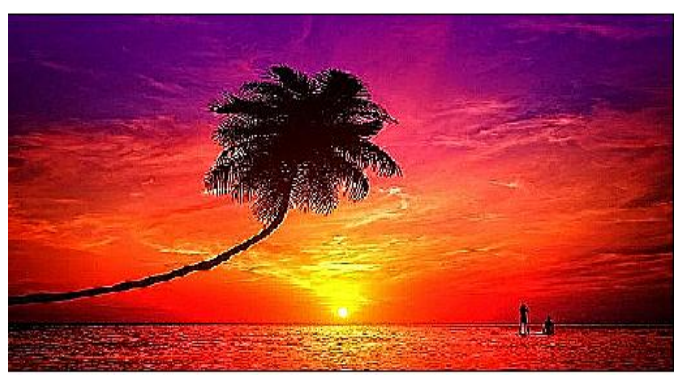

(b)

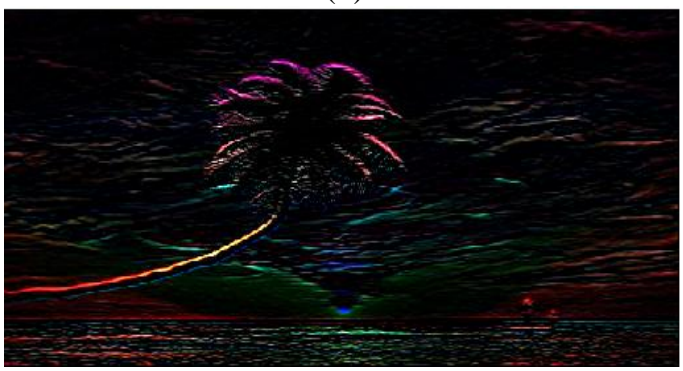

(d)

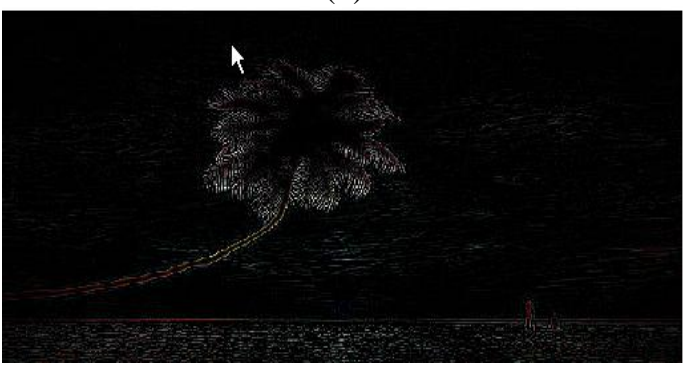

(f)

Figure 1 Smoothing results: a) Original image b) Corrupted image c) SSA d) PSA e) ASA, and f) LSA

The isometric plots can provide the display of a 3D visualization [24]. It is drawn in a shaded graphic or a 4 wire mesh. The concerning images are plotted as presented in Figure 2. The plot is important for 
understanding the frequency domains and their mathematical distribution function. In MATLAB correlations can be calculated with the function which reflects the frequency domain. Note that to find the discrete function, we have to reverse the arguments and swap the end of the smaller input with zeros in order that they hold the identical length. As MATLAB cannot handle negative or zero arguments the correlation sample with zero figures is the central

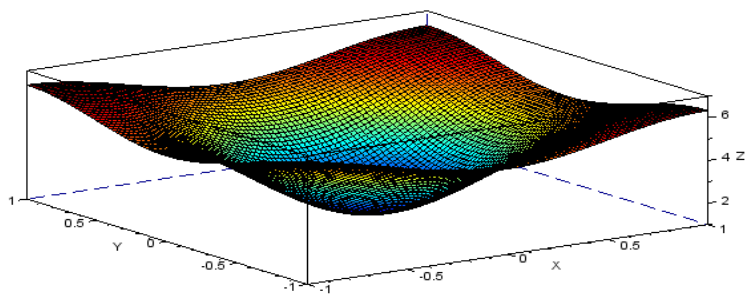

(a)

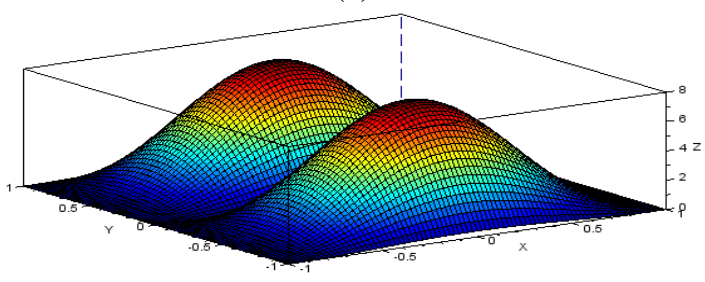

(c)

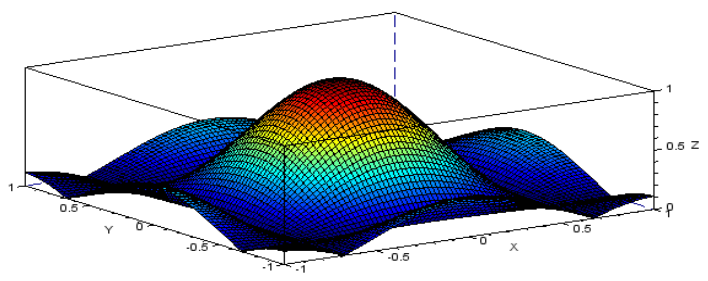

(e) component of the output vector. An alternating method of finding the correlation without stuffing with zeros is extending to the convolution vector computation. A benefit of the isometric plot is that it can point out a worthy perception of the intensity function as a scale is indicated on the $x-y-z$ axes. It is ineffective for the image indication alone with no isometric representation.

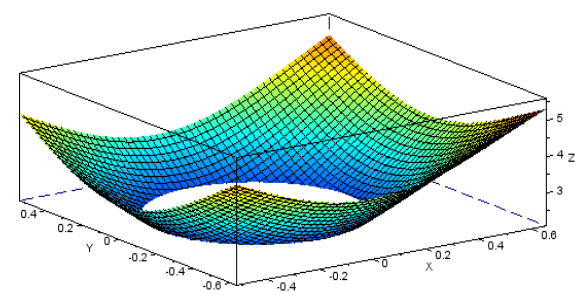

(b)

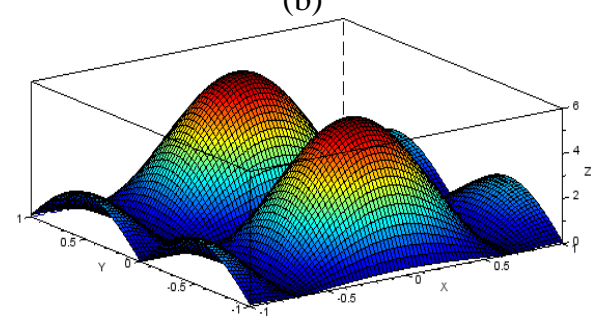

(d)

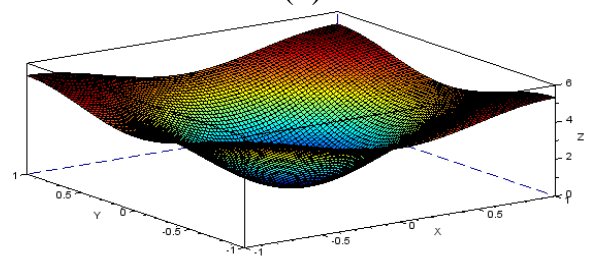

(f)

Figure 2 Frequency visualization: a) Original image b) Corrupted image c) SSA d) PSA e) ASA, and f) LSA

Huge effort and computational time are spent on computing the descent of any windows as smoother takes every pixel in the image, and then values are estimated. Digital images enclose the total numeric model, then they can be definitely represented by histogram [25]. We create the histogram of concerning the images as depicted in Figure 3 which is supportive in understanding the feature of pixels. Histogram of digital images represents a graphical distribution model, then it is applied to the digital camera. Professional photographers employ these graphics as advice to decide about the adjustment of tones, and whether the image information will be affected by unexpected shadow. Image quality improvement is essential in image analytics. It is to enhance the 5 quality of blurred images by amplifying the intensity varies of considered background and the whole objects. Histogram thus is critical for both image analytics and improvement.

\section{Conclusion}

Our research contributes a comparable approach to practitioners who are operating in distinct areas and from dissimilar experiences. The comprehension of image analyzers is intensified, and their time spent is diminished as they can see an isometric or surface plot. It encourages them to concentrate on the perception rather than the complex mathematics and variables. Image smoothing algorithms can be efficiently employed with a less time complexity of 
calculation per pixel. By doing the evaluation between image smoothing algorithms, we settle that ASA technique outperforms other approaches regarding noise filtering as it focuses on static window size and computes the edges accordingly.
Not to mention it is simply carried out. Future researches include the optimization approach like Gradient Descent Algorithm (GDA) and a computer vision with mobile objects.

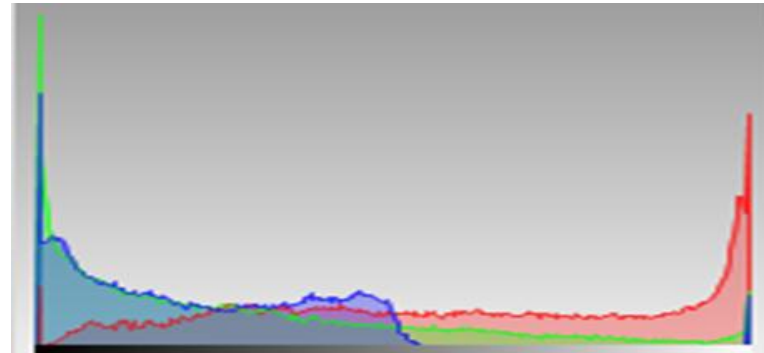

(a)

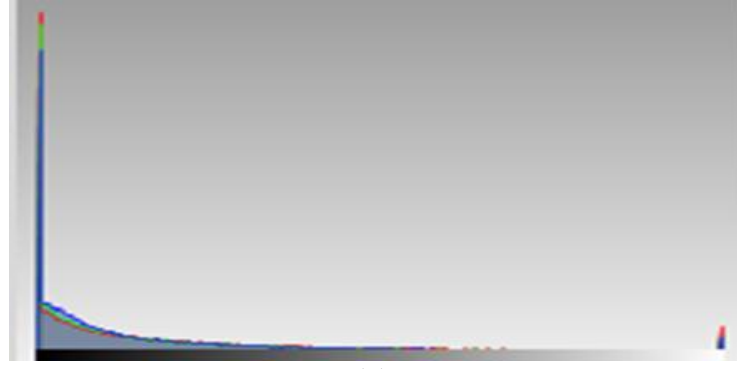

(c)

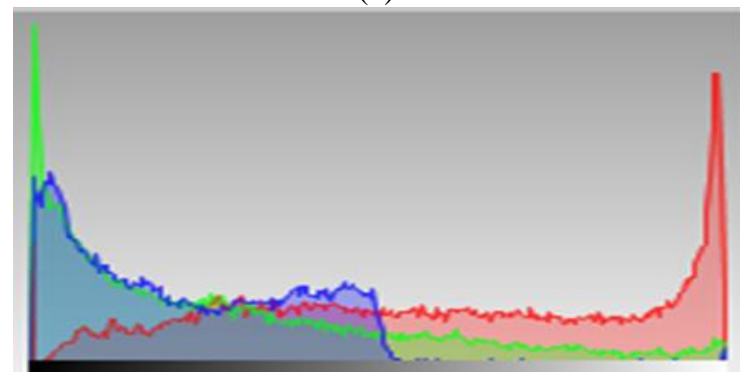

(e)

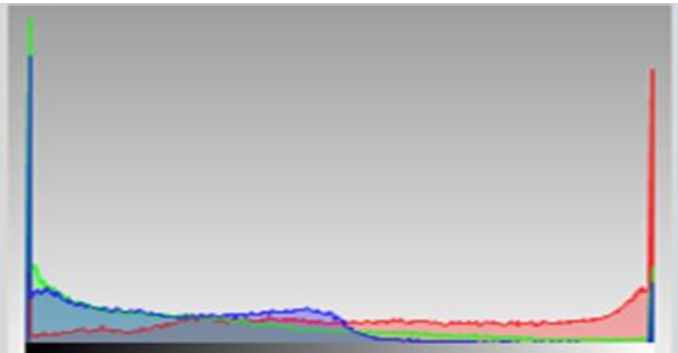

(b)

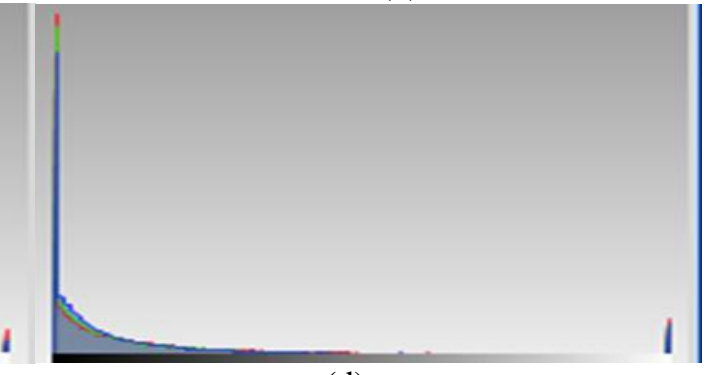

(d)

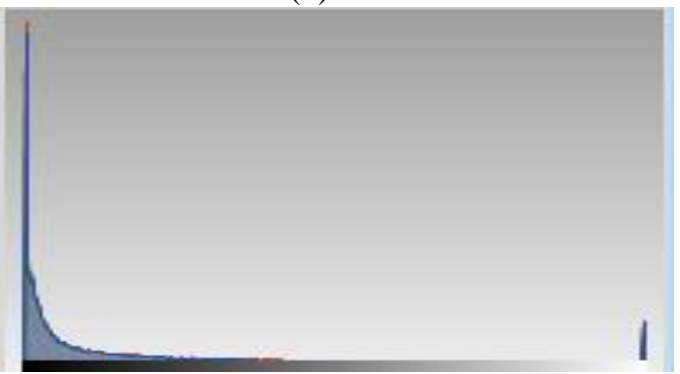

(f)

Figure 3 Histogram: a) Original image b) Corrupted image c) SSA d) PSA e) ASA, and f) LSA

\section{Acknowledgment}

None.

\section{Conflicts of interest}

The authors have no conflicts of interest to declare.

\section{References}

[1] Gunawan TS, Gani MH, Rahman FD, Kartiwi M. Development of face recognition on raspberry pi for security enhancement of smart home system. Indonesian Journal of Electrical Engineering and Informatics (IJEEI). 2017; 5(4):317-25.

[2] Patel N, Shah A, Mistry M, Dangarwala K. A study of digital image filtering techniques in spatial image processing. In proceedings of the international conference on convergence of technology 2014 (pp. 1$6)$.
[3] Rashid MM, Musa A, Rahman MA, Farahana N, Farhana A. Automatic parking management system and parking fee collection based on number plate recognition. International Journal of Machine Learning and Computing. 2012; 2(2):93-8.

[4] Vasanth PC, Nataraj KR. Facial expression recognition using SVM classifier. Indonesian Journal of Electrical Engineering and Informatics. 2015; 3(1):16-20.

[5] Penangsang O, Soeprijanto A. Matlab/simulink simulation of unified power quality conditionerbattery energy storage system supplied by PV-wind hybrid using fuzzy logic controller. International Journal of Electrical \& Computer Engineering. 2019; 9(3):1479-95.

[6] Anjum MA. A new approach to adaptive signal processing. arXiv preprint arXiv:1504.06054. 2015.

[7] Eftekhari S, Sadegh MO. The effect of load modelling on phase balancing in distribution networks using 
search harmony algorithm. International Journal of Electrical \& Computer Engineering. 2019; 9(3):146171.

[8] Halimi A, Mailhes C, Tourneret JY. Nonlinear regression using smooth Bayesian estimation. In international conference on acoustics, speech and signal processing 2015 (pp. 2634-8). IEEE.

[9] Chouchane M, Paris S, Le Gland F, Musso C, Pham DT. On the probability distribution of a moving target. Asymptotic and non-asymptotic results. In international conference on information fusion 2011 (pp. 1-8). IEEE.

[10] Bennasar M, Hicks Y, Setchi R. Feature selection using joint mutual information maximisation. Expert Systems with Applications. 2015; 42(22):8520-32.

[11] Al-Aboosi YY, Sha'ameri AZ. Improved signal denoising in underwater acoustic noise using $\mathrm{S}$ transform: a performance evaluation and comparison with the wavelet transform. Journal of Ocean Engineering and Science. 2017; 2(3):172-85.

[12] Jung M, Marquina A, Vese LA. Variational multiframe restoration of images degraded by noisy (stochastic) blur kernels. Journal of Computational and Applied Mathematics. 2013; 240:123-34.

[13] ElSaid WK. Watermarking digital artworks. International Journal of Computer Applications. 2015; 125(12):17-23.

[14] Rani V. A brief study of various noise model and filtering techniques. Journal of Global Research in Computer Science. 2013; 4(4):166-71.

[15] Nazir I, Jan R, Nazir U, Yousuf A. A comparative review paper for noise models and image restoration techniques. International Journal of Computer Science and Mobile Computing. 2017; 6(6):405-10.

[16] Garg R, Kumar A. Comparison of various noise removals using Bayesian framework. International Journal of Modern Engineering Research. 2012; 2(1):265-70.

[17] Praveen KS, Hamarnath G, Babu KP, Sreenivasulu M, Sudhakar K. Implementation of image sharpening and smoothing using filters. International Journal of Scientific Engineering and Applied Science. 2016; 2(1):7-14.

[18] Das S, Saikia J, Das S, Goni N. A comparative study of different noise filtering Techniques in digital images. International Journal of Engineering Research and General Science. 2015; 3(5):180-90.
[19] Gupta S, Mazumdar SG. Sobel edge detection algorithm. International Journal of Computer Science and Management Research. 2013; 2(2):1578-83.

[20] Alshamarti HA. Removal of gaussian noise on the image edges using the prewitt operator and threshold function technical. Journal of Computer Engineering. $2013 ; 15: 81-5$.

[21] Gupta G. Algorithm for image processing using improved median filter and comparison of mean, median and improved median filter. International Journal of Soft Computing and Engineering. 2011; 1(5):304-11.

[22] Dhar R, Gupta R, Baishnab KL. An analysis of CANNY and LAPLACIAN of GAUSSIAN image filters in regard to evaluating retinal image. In international conference on green computing communication and electrical engineering 2014 (pp. 16). IEEE.

[23] Goyal A, Bijalwan A, Chowdhury MK. A comprehensive review of image smoothing techniques. International Journal of Advanced Research in Computer Engineering \& Technology. 2012; 1(4):315-9.

[24] Fan Z, Weng Y, Chen G, Liao H. 3D interactive surgical visualization system using mobile spatial information acquisition and autostereoscopic display. Journal of Biomedical Informatics. 2017; 71:154-64.

[25] Kaur H, Sohi N. A study for applications of histogram in image enhancement. The International Journal of Engineering and Science. 2017; 6(6):59-63.

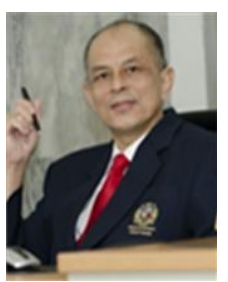

Chanintorn Jittawiriyanukoon received the B.Eng degree in electrical engineering from Kasetsart University, Bangkok, Thailand in 1984, M.Eng. and D.Eng. degrees in data communication from Osaka University, Osaka, Japan in 1987 and 1990 respectively. $\mathrm{He}$ is presently an Assistant Professor in Information Technology and also working as a Dean of Graduate School of Advanced Technology Management, Assumption University, Samut Prakan Province, Thailand. His research focuses include Big Data Curation, Computer Vision, Fast Routing Algorithm, Adaptive Rate Control (ARC) Algorithm, Queuing Network Analysis and High-Speed-Network Performance Evaluation.

Email: chanintornjtt@au.edu 